\title{
Nuevas narrativas periodísticas entre la información y la simulación lúdica: los docuwebs y los newsgames
}

\section{Eva Herrero-Curiel ${ }^{1}$ Antonio José Planells de la Maza²}

Recibido: 01/02/2019

Aprobado por pares: 11/06/2019
Enviado a pares: 01/02/2019

Aceptado: 17/06/2019

DOI: $10.5294 /$ pacla.2020.23.2.5

Para citar este artículo / to reference this article / para citar este artigo Herrero-Curiel, E. y Planells de la Maza, A. J. (2020). Nuevas narrativas periodísticas entre la información y la simulación lúdica: los docuwebs y los newsgames. Palabra Clave, 23(2), e2325. https://doi.org/10.5294/pacla.2020.23.2.5

\section{Resumen}

Este artículo tiene por objetivo analizar cómo cooperan el periodismo digital y la cultura del videojuego moderno en dos formas de simulación lúdica e inmersión periodística: los docuwebs y los newsgames. Para ello, se ha establecido una metodología de análisis cualitativo con una doble perspectiva: el nivel del discurso informativo-periodístico (es decir, los contenidos y sus formas de proyección) y a nivel de retórica procedural (esto es, a través de las mecánicas de juego) que se aplica a dos casos de estudio de RTVE Lab: el newsgame MonteLab y el docuweb Guerra a la mentira. Los principales resultados muestran cómo el diseño de MonteLab establece un diálogo entre un rico sistema de fuentes periodísticas y unas mecánicas de juego que habilitan la toma de decisiones ante los problemas sociales de la actualidad. En una línea similar, el docuweb Guerra a la mentira establece un conjunto de casos reales y usa la verificación de la información y las fake news como motor periodístico sobre el que construir una propuesta lúdi-

$\bowtie$ https://orcid.org/0000-0003-0801-2519. Universidad Carlos III de Madrid, España. eherrero@hum.uc3m.es

https://orcid.org/0000-0001-7396-2751. Universidad Pompeu Fabra, España. aplanells@tecnocampus.cat 
ca de resolución de puzles e impulso narrativo. Por todo ello, y a modo de conclusiones, podemos comprobar que el newsgame contemporáneo busca empoderar al jugador como un ciudadano informado que explota las dinámicas lúdicas para proveer soluciones sociales basadas en informaciones periodísticas. Por otro lado, el docuweb supera el estaticismo hipertextual para adentrarse en dinámicas más complejas y diseños lúdicos más inmersivos gracias a la superación de la mera descripción periodística de la realidad.

\section{Palabras clave (Fuente: tesauro de la Unesco)}

Nuevas narrativas; periodismo; docuweb; newsgame; transmedia. 


\section{New Journalistic Narratives between Information and Playful Simulation: Docuwebs and Newsgames}

\section{Abstract}

This article aims to discuss how digital journalism and the culture of modern videogames interface in two forms of playful simulation and journalistic immersion: docuwebs and newsgames. For this, a qualitative analysis method has been used with a double perspective: the level of informativejournalistic discourse (i.e., contents and their projections) and the level of procedural rhetoric (i.e., through game mechanics). This is applied to two RTVE Lab case studies: newsgame MonteLab and docuweb Guerra a la mentira. The main results show how the design of MonteLab opens a dialogue between a rich journalistic source system and some game dynamics that enable decision-making regarding today's social problems. Likewise, Guerra a la mentira offers a set of real cases and uses fact-checking and fake news as the journalistic foundations on which to build a playful proposal for puzzle solving and narrative momentum. In conclusion, we could verify that the contemporary newsgame seeks to empower the player as an informed citizen who takes advantage of playful dynamics to provide social solutions based on journalistic information. At the same time, the docuweb goes beyond hypertextual staticism and the mere journalistic description of reality to get into more complex dynamics and more immersive playful designs.

\section{Keywords (Source: Unesco Thesaurus)}

New narratives; journalism; docuweb; newsgame; transmedia. 


\section{Novas narrativas jornalísticas entre a informação e a simulação lúdica: os docuwebs e os newsgames}

\section{Resumo}

Este artigo tem como objetivo analisar como o jornalismo digital e a cultura do videogame moderno cooperam em duas formas de simulação lúdica e imersão jornalística: os docuwebs e os newsgames. Para isso, foi estabelecida uma metodologia de análise qualitativa com uma dupla perspectiva: a do discurso informativo-jornalístico (isto é, os conteúdos e suas formas de projeção) e a de retórica procedural (ou seja, por meio das mecânicas de jogo), que é aplicada a dois casos de estudo de RTVE Lab: o newsgame MonteLab e o docuweb Guerra a la mentira. Os principais resultados mostram como o desenho de MonteLab estabelece um diálogo entre um rico sistema de fontes jornalísticas e mecânicas de jogo que possibilitam a tomada de decisões diante dos problemas sociais da atualidade. Numa linha semelhante, o docuweb Guerra a la mentira estabelece um conjunto de casos reais e usa a verificação da informação e das fake news como motor jornalístico sobre o qual construi uma proposta lúdica de resolução de quebra-cabeça e impulso narrativo. Por tudo isso e como conclusão, podemos comprovar que o newsgame contemporâneo busca empoderar o jogador como um cidadão informado que explora as dinâmicas lúdicas para dar soluções sociais baseadas em informações jornalísticas. Por sua vez, o docuweb supera o estaticismo hipertextual para entrar em dinâmicas mais complexas e desenhos lúdicos mais imersivos graças à superação da mera descrição jornalística da realidade.

\section{Palavras-chave (Fonte: tesauro da Unesco)}

Novas narrativas; jornalismo; docuweb; newsgame; transmídia. 


\section{Introducción: narrativas periodísticas emergentes en la web social}

Las nuevas formas de relato digital, los múltiples canales de difusión, así como la participación activa de los usuarios son variables inherentes al ejercicio periodístico. En la actualidad, no se puede concebir ningún género informativo sin tener en cuenta las características de internet y las nuevas formas de consumo que marcan el storytelling a través del que se difunden los contenidos, sean estos de naturaleza informativa o no. Además, la convergencia tecnológica por la que atraviesan los medios de comunicación desde que entramos en el siglo XXI ha llevado a que los relatos sean presentados a partir de una narración transmediática que se desarrolla a través de múltiples plataformas; en ellas cada nuevo texto hace una contribución específica y valiosa a la totalidad, donde cada medio hace lo que se le da mejor (Jenkins, 2008, p. 101). Las historias se conciben desde una perspectiva transmedia para que el usuario final las consuma de diversas maneras. Una de estas nuevas formas de consumo es también desde el diseño de experiencias lúdicas disponibles en múltiples formatos y plataformas. Por ello, el reto del periodismo en su dimensión digital es particularmente relevante: ¿cómo se puede conciliar un discurso informativo y fiable en el contexto transmediático y que asuma además la presencia del videojuego como un medio particularmente relevante para el usuario contemporáneo? Así pues, esta investigación parte de este problema para analizar cómo dos fenómenos mediáticos informativo-lúdicos, el newsgaming y el docuweb, consiguen trazar puentes entre periodismo y juego. Para ello, y en primer lugar, resulta conveniente definir y delimitar el alcance de algunos de los principales conceptos: transmedialidad, redes sociales y storytelling digital, newsgaming y docuweb.

\section{Transmedialidad, redes sociales y storytelling digital}

Industrias como la del cine, la televisión, la publicidad o los videojuegos han encontrado en esta nueva forma de narrar el camino para llegar a diferentes públicos. Jenkins (2008, p. 102) considera que una buena franquicia transmediática trabaja para atraer a múltiples públicos introduciendo los conte- 
nidos de una manera algo distinta en diferentes medios. El hecho de que el receptor sea ahora un usuario activo hace que el relato se haya transformado y dado lugar a un "remix narrativo" (Ferrari, 2010) que utilizan los medios para difundir sus contenidos.

A pesar de que Jenkins (2008) es considerado como uno de los precursores del término transmediático, otros autores como Moloney (2011) han partido de su postulado para extrapolar estas características al ámbito puramente periodístico. El autor parte de la idea de que las narrativas transmediáticas que utilizan los periodistas contienen un importante componente social en el que el objetivo principal radica en llegar al mayor número de personas posibles.

Ryan (2004) considera que el medio digital afecta a la narrativa en tres aspectos relacionados con sus diferentes niveles. A nivel pragmático, se ofrecen nuevas formas para que los usuarios participen en la narración, a través de intercambio de historias en tiempo real o participando en la creación colectiva de historias. A nivel discursivo, se producen nuevos modos de presentar la historia que requieren del usuario nuevas estrategias interpretativas. Y, finalmente, en el nivel semántico, no se trata de una nueva lógica, pero es necesario encontrar una equilibrio correcto entre el medio, la forma y la sustancia del mensaje (p. 354).

No solo las características de la web 2.0 (interactividad, participación, transmedialidad, viralización, etc.) influyen en la forma de presentar las noticias, o cualquier otro género informativo, sino que también el patrón de consumo del usuario determina la forma final que adopta el relato. Es en este punto donde se esboza un nuevo concepto como es el de la inmersión. Esta se plantea como una nueva forma de consumo en la que el usuario se introduce en un "pseudoambiente", en palabras de Lippman (2003), donde la experiencia del receptor está mediada.

Por su parte, las redes sociales en el contexto periodístico enriquecen la transmedialidad, ya que con ellas se ha generado otra forma de narración. Una narración más interactiva y también con ciertas peculiaridades. 
Así, por ejemplo, el periodista que decide contar una historia a través de Twitter debe adaptarse a los límites narrativos que este le impone; sin embargo, sus tuits pueden integrarse en la noticia "tradicional" escrita para la web, para el papel, locutada para radio o transmitida por televisión (Herrero, 2014). De esta forma, cualquier persona puede interrumpir su lectura para consultar la conversación generada en Twitter y hacerlo de forma simultánea a través de cualquier dispositivo.

El storytelling digital permite a los profesionales, que hoy en día trabajan con las nuevas tecnologías, crear historias que integren tanto lo online como lo offline y donde el periodista puede desencadenar el proceso, pero los usuarios contribuyen a su desarrollo y acaso transformación (Carrera, Limón, Herrero y Sainz de Baranda, 2013). El patrón de consumo del usuario determina la forma final que adopta el relato. Hoy más que nunca el receptor asume un papel activo dentro de una historia generada para él.

Los medios de comunicación y los nuevos perfiles profesionales que han emergido dentro del mundo periodístico apuestan, cada vez más, por la experimentación y creación de formatos alternativos a los tradicionales. Formatos que puedan adaptarse a la ruptura que internet ha provocado en el tiempo y en el espacio. En España, es la televisión pública, RTVE, la que está apostando firmemente por la adaptación de géneros informativos audiovisuales a la narrativa web, a través del Lab. Esta unidad experimenta con la creación de nuevos proyectos periodísticos que rompen con los cánones tradicionalmente establecidos cuyo objetivo es "hacer frente a los retos de futuro y poner pie en pared a una crisis que no desiste en su empeño de robar lectores y descapitalizar la industria periodística" (López y Ufarte, 2016, p. 13).

\section{Una aproximación conceptual al newsgame: entre el relato informativo y la experiencia lúdica}

En el actual contexto digital marcado por la interactividad y la información, emerge el concepto de newsgames, "videojuegos basados en noticias [...], o lo que podemos considerar como fusión entre la simulación lúdica y aspectos relevantes de la actualidad" (Martínez, 2016, p. 161). Aplicado por 
primera vez por el diseñador Gonzalo Frasca en el juego September 12th (2003) y propuesto como categoría propia dentro de los game studies por Bogost, Ferrari \& Schweizer (2012), la noción de newsgame nos remite a la producción de experiencias lúdicas con la intención de participar en la esfera pública con argumentos que ilustren las distintas perspectivas de las noticias (Sicart, 2008, p. 30). De este modo, la tipología de videojuegos newsgame supera la mera función lúdica del ocio digital y se inscribe en un tipo de juego político que pretende, mediante la interacción entre un usuario y un mundo de ficción constituido por hechos noticiosos, desplegar un posicionamiento informado de la realidad. Así, los jugadores de newsgames no deben entenderse como consumidores, sino como ciudadanos que comparten una visión de interés público (p. 3).

En España, los newsgames ya han empezado a estudiarse tanto como objetos periodísticos (Gómez y Cabeza, 2016; Gómez y Navarro, 2013) como en conexión con otros fenómenos emergentes, por ejemplo, los procesos de gamificación (Martínez, 2016). En cambio, para otros autores, este género es un híbrido entre "periodismo, editorial y juego, y que se caracterizan por la interacción que tiene como objetivo estimular en el participante una emoción y/o una reflexión sobre el tema en cuestión" (Celeste, 2008, p. 158). Este doble objetivo se encuentra también en la definición de juego serio, aquellos que no se dirigen solo al entretenimiento (algo parecido a lo que ocurre con las soft news y hard news), sino que pretenden transmitir datos (informar, enseñar), sensibilizar (estimular a la acción, involucrar) y dar sentido (contextualizar, editorializar, explicar (Samson, 2015, p. 124).

Del mismo modo que se habla de web 3.0 en alusión a la siguiente fase de la web social, en los últimos años parece que los newsgames avanzan hacia formas más complejas, determinadas por tecnologías como la realidad virtual (Jones, 2017). Es en este contexto en el quesurge el periodismo inmersivo.

Hoy en día, existen pocos estudios en el área de la comunicación en España que aborden este tipo de periodismo. Desde la década de 1990, diversos grupos de investigación en los Estados Unidos comenzaron a inves- 
tigar y experimentar con las posibilidades de aplicar la lógica del videojuego al contenido informativo (Domínguez-Martín, 2015).

Domínguez-Martín (2015) define el periodismo inmersivo como "una forma narrativa que busca la inmersión a través de técnicas interactivas y visuales consistentes en fomentar el rol activo del usuario en el relato y una experiencia sensorial de exploración del espacio" (p. 420), definición que pone el foco de atención en la narrativa transmedia y en el concepto de interactividad.

\section{El docuweb como nuevo género periodístico}

Mientras que el newsgame no es un género muy explotado a día de hoy en los entornos periodísticos, los docuwebs sí parecen consolidarse poco a poco como nuevos géneros de no ficción, fruto de una hibridación entre narraciones más tradicionales y lineales (el documental clásico) y las múltiples posibilidades narrativas que ofrece internet. El docuweb queda definido como "una forma emergente, pendiente de exploración y de delimitación, y fruto de una doble hibridación: entre audiovisual — género documental— e interacción —-medio digital interactivo-, y entre información — contenidos_y entretenimiento —interfaz navegable_-" (Gifreu, 2013, p. 148).

El docuweb nace como resultado de la exploración de nuevos formatos periodísticos capaces de explotar todos los recursos que ofrece la web hoy en día. Esta exploración y creación nace en laboratorios de corte informativo que tienen una vocación investigadora e innovadora dentro de un contexto en constante cambio. En España, el laboratorio pionero en este género es el Lab de RTVE, pero fuera de nuestras fronteras encontramos laboratorios similares en medios como The Guardian (Reino Unido), The New York Times (EE. UU.) o la BBC (Reino Unido). Por tanto, existe una vinculación importante entre el docuweb y la profesión periodística.

Estos laboratorios nacen con la vocación de encontrar nuevas formas de relato que atraigan más audiencia. Una audiencia cada vez más digital, diversificada y que consume a través de múltiples dispositivos. Receptores que son multitarea y que apenas pueden seguir relatos audiovisuales que 
duren más de dos minutos. Una realidad que responde a la filosofía 2.0: contenidos cortos y fáciles de viralizar.

En el docuweb, se encuentran características propias de otros géneros periodísticos como el reportaje o el documental, sin embargo, lo que convierte el docuweb en un género independiente es su carácter interactivo. Cualquier proyecto que comienza con la intención de documentar lo real y que utilice tecnología digital interactiva para realizarlo puede ser considerado como un documental interactivo (Aston \& Gaudenzi, 2012, p. 125).

El usuario no se limita a consumir información de forma pasiva o lineal, más bien adopta un rol activo durante el consumo del mensaje (Martínez y Díaz, 2016, p. 72). El consumidor navega a través de distintos elementos multimedia troceados para que sea el usuario el que decida cuándo y cómo consumirlo, variables que definen el consumo informativo en internet.

De esta forma, el docuweb es el formato que más se está utilizando a nivel informativo con la finalidad de acercar un hecho noticioso y de interés público a la sociedad. Este nuevo género aúna información, interpretación y concienciación ciudadana acerca de realidades que pueden pasar inadvertidas en la agenda mediática tradicional, monopolizada por la política nacional o la información institucional. De ahí que la mayoría de los docuwebs que encontramos traten temas bélicos, económicos o fenómenos que constituyen tendencias a lo largo de los años.

\section{Metodología}

\section{Análisis de contenido en textos periodísticos}

Desde el punto de vista periodístico, se ha aplicado el análisis de contenido cualitativo a MonteLab y Guerra a la mentira. Para ello, se ha partido de la definición de análisis de contenido revisada por Bardin quien señala que el objetivo de esta técnica de investigación es "obtener indicadores (cuantitativos o no) por procedimientos sistemáticos y objetivos de descripción de contenidos de los mensajes, permitiendo la inferencia de co- 
nocimientos relativos al contexto social de estos mensajes" (1996, p. 32). En esta investigación, el análisis de contenido ha sido aplicado a dos casos de estudio ateóricos, íntegramente descriptivo y en el que no hay un interés de realizar generalizaciones ni de formular hipótesis a posteriori (Kazez, 2009). El primer paso en esta investigación ha sido determinar el objeto de análisis para establecer, a partir de una primera observación exhaustiva, una serie de categorías en las que se encuadran las principales características que debe tener un mensaje periodístico. ${ }^{3} \mathrm{~A}$ continuación, se han añadido otras variables de estilo formal y que responden a la idiosincrasia del medio. El análisis se ha completado con la revisión de los documentos originales facilitados por los creadores de ambos proyectos y que ayudan a entender las condiciones de producción o recepción del texto. Estas fuentes de información primarias han sido los guiones originales de ambos proyectos (literario y técnico) y los documentos utilizados para construir las tramas.

\section{La retórica procedural y el modelo MDA (mechanics, dynamics, aesthetics)}

Bogost (2007) propuso el concepto de procedural rethoric o retórica de procesos (o procedural) como marco de comprensión de aquellos videojuegos que, junto con su vocación eminentemente lúdica, plantean discursos complementarios de índole ideológica o informativa y que puede definirse como la práctica interpretativa de argumentaciones a través de procesos (p. 28). Partiendo de la noción clásica de retórica por la que "se describen los procedimientos de utilización del lenguaje (escrito, visual, etc.) con una finalidad persuasiva o estética" (Gómez y Navarro, 2013, p. 36), Bogost aplica la noción procedural de Murray (1998) para considerar que los videojuegos emergen como una excepción ontológica a los medios retóricos tradicionales. Así, la dimensión retórica del videojuego se encuentra en la forma en la que los argumentos se insertan en las reglas del juego y en cómo las reglas se expresan, comunican y son entendidas por el jugador (Sicart, 2011, p. 3). La popularidad de la retórica de procesos ha crecido enormemente en los últimos años, con múltiples trabajos e investigaciones que analizan

3 Estas categorías se han recogido en una ficha de análisis que puede consultarse en el anexo 1. 
la construcción discursiva del juego digital a través de, precisamente, el carácter sistémico y procedural de las reglas del juego (Brathwaite \& Sharp, 2010; Flanagan, 2009).

Asumiendo la centralidad procedimental y retórica de las reglas de juego de los newsgames, en esta investigación adoptamos un modelo metodológico específico denominado MDA (mechanics, dynamics, aesthetics) que nos será útil para segmentar los títulos de la muestra en distintos niveles de análisis procedural.

La teorización del MDA fue realizada por Hunicke, Leblanc \& Zubek (2004, p. 2) y concibe el juego como un artefacto diseñado, perspectiva que ayuda a enmarcar los juegos como sistemas que construyen comportamientos a través de la interacción y que permite dividir o separar el juego en tres categorías: mecánicas, dinámicas y estética. En función de la perspectiva, es decir, bien sea la intención creadora del diseñador, bien sea la visión de consumo del jugador, los componentes se experimentan de manera distinta. Así, la perspectiva del diseñador siempre partirá del núcleo más abstracto de la experiencia lúdica (las reglas o mecánicas) para configurar un sistema que, en la interacción con el usuario (estrategias de juego o dinámicas), hará emerger un tipo de emoción o experiencia estética más o menos prevista. En sentido contrario, el usuario final o jugador percibe el videojuego como un sistema audiovisual que proyecta, mediante la experiencia de juego, un conjunto emocional o constructo estético. La iteración en dicha experiencia hace que el jugador entienda el juego como un sistema y, de este modo, racionaliza un conjunto de prácticas lúdicas que lo llevan, irremediablemente, a establecer cuáles son las mejores o más óptimas estrategias de juego, también llamadas dinámicas. Finalmente, el mayor grado de experiencia del usuario final en su relación con la creación digital lúdica conlleva el cálculo en su nivel más abstracto, es decir, el conocimiento profundo de las particularidades y principales formas de funcionamiento de las mecánicas o reglas específicas del sistema. A partir de esta doble visión (la creadora del diseñador, de lo abstracto a lo emocional, y la del usuario, de lo emocional a la comprensión normativa), se pueden conceptualizar de manera clara los principales componentes del modelo: las mecánicas, las dinámicas y la estética. 
Así, para Hunicke et al. (2004, p. 2), las mecánicas de juego configuran la lógica del sistema, sus condiciones de victoria y, en general, todas aquellas normas y limitaciones que suelen inscribirse a nivel algorítmico. De este modo, las mecánicas conforman una macrocategoría que contiene todos elementos de diseño de juegos que conocemos como reglas en su doble acepción como normas constitutivas (a nivel más abstracto y vinculadas al sistema lógico-matemático del juego) o normas operativas (reglas orientadas al funcionamiento del juego y que son facilitadas a los jugadores normalmente mediante tutoriales o en el manual de juego) (Salen \& Zimmerman, 2004, p. 139).

Tras ellas, las dinámicas determinan el comportamiento en tiempo real del sistema a partir de las distintas acciones del usuario (Salen \& Zimmerman, 2004, p. 2). De este modo, mientras que las mecánicas proponen un juego como sistema estático, las dinámicas son en realidad la puesta en acción de dicho modelo, y así consignan las acciones del usuario y hacen emerger las distintas estrategias de juego.

Finalmente, el componente estético describe las reacciones emocionales del jugador según las previsiones establecidas por el sistema (Salen \& Zimmerman, 2004, p. 2). Más allá del criterio estrictamente estético-visual (gráficos, transiciones o animaciones, entre otras manifestaciones) o auditivo (efectos sonoros o banda sonora), la concepción estética del MDA se vincula, pues, con una expectativa emocional establecida por el diseñador y, hasta cierto punto, esperada en el usuario. Así, en el modelo MDA, las tres capas o manifestaciones del juego como artefacto deben cooperar entre sí: determinadas mecánicas que prefiguran un sistema lógico deben manifestar, en el nivel de dinámicas, unas acciones estratégicas del jugador para provocar, en lo estético, un conjunto emocional buscado.

De este modo, en el análisis de casos, segmentaremos cada uno de los títulos a partir de la tríada MDA para entender cómo funciona y cómo puede impactar la retórica de procesos este triple nivel: en las reglas de juego, en las formas de jugar y en las principales reacciones esperadas en el jugador. 


\section{Análisis de casos}

\section{MonteLab: el reto de ser propietario}

Se trata de un newsgame que en 2014 lanza el equipo del Lab de RTVE.es con el objetivo de completar una serie de documentales, con una narración lineal, emitidos en el programa Documentos TV sobre la burbuja inmobiliaria bajo el título de "Casas vacías" y "Las nuevas ruinas". El proyecto de MonteLab ofrece al usuario la posibilidad de consumir de forma transmedia el contenido informativo que constituye el core de los documentales, además la saturación informativa sobre el asunto lleva a buscar otras formas de contar la historia. El plus en esta ocasión es que el espectador se sumerge en un escenario ficticio que simula al real y en el que tiene que adoptar el rol de propietario.

El primer componente periodístico que se encuentra en MonteLab es el tema que cubre (el incremento del negocio inmobiliario y la construcción de infraestructuras) y que responde a los criterios de noticiabilidad que cualquier género informativo debe contener:

- Hechos: en este caso, se trataría de la construcción de viviendas por encima de la renta disponible, el incremento del precio de los terrenos, las operaciones de reclasificación de suelo, las ayudas al pago de alquiler, las hipotecas excesivas o el mercado altamente especulativo.

- Actualidad: el juego nace en 2014, y aunque está ambientado entre 2007 y 2012, la percha de actualidad está justificada al tratarse de un tema abierto y que continúa en el presente.

- Repercusión social: el acceso a una vivienda digna, los desahucios o las demandas por la cláusula suelo siguen teniendo un fuerte impacto entre los ciudadanos y generan debate en los medios de comunicación.

- Proximidad: se trata de un tema que directa o indirectamente afecta a un número importante de familias españolas.

- Conflicto: se genera una importante tensión entre varios actores sociales. Numerosas familias pierden su vivienda al no poder afrontar la hipoteca. 
- Impacto: repercusión a nivel social, económico y político.

- Interés humano: detrás de los datos y las cifras que se presentan en el newsgame hay historias y testimonios de ciudadanos reales que se han visto afectados por este fenómeno.

El segundo componente periodístico, a nivel de contenido, es el tratamiento de las fuentes informativas. Del mismo modo que ocurre en un reportaje o en un documental, MonteLab se apoya en un importante número de fuentes de diversa naturaleza que otorgan al relato mayor credibilidad y pluralidad. Fuentes documentales (53\%), ciudadanas (21\%), institucionales $(16 \%)$ y expertas $(10 \%)$ emergen a modo de totales con una duración que en ningún caso supera el minuto o minuto y medio. Su testimonio sirve para ilustrar y argumentar cada una de las decisiones que el jugador tiene que tomar durante el transcurso de la partida.

Las fuentes documentales presentan datos y cifras reales de los diferentes años en los que transcurre la trama del newsgame. Esta información es presentada mediante infografías o gráficos con el fin de visualizar macrodatos que permitan al usuario contextualizar mejor el tema tratado. Los textos que aparecen en MonteLab están constituidos por titulares informativos que corresponden a noticias publicadas durante esas fechas y que sirven de guía al jugador en cada nueva etapa.

A nivel formal, los principales elementos que aparecen durante el transcurso del juego son vídeos (22), gráficos (6) y audios (2), acompañados de textos. Todos los elementos multimedia que aparecen constituyen de forma independiente piezas puramente periodísticas de un marcado carácter inmersivo. Los vídeos son entrevistas con las fuentes, los gráficos o infografías son visualizaciones de macrodatos que permiten contextualizar mejor el tema tratado, los textos están constituidos por noticias reales publicadas en prensa durante ese transcurso de tiempo y titulares que sirven de guía al usuario en cada nueva etapa. De forma sucinta, también hay espacio para audios radiofónicos en los que hablan diversas fuentes. Estos elementos que aparecen al principio o al final de cada bloque narrativo dentro del juego ayudan al usuario a conocer información complementaria sobre el asunto. 
Desde la perspectiva del análisis ludológico, MonteLab tiene un marcado tono sarcástico en el que prima la estructura narrativa ramificada o arbórea propia del género de aventuras. En concreto, utiliza modelos de elección textual en la línea de la ficción interactiva mientras que la ausencia de puzles u otras formas de impulso narrativo lo alejan del otro gran subgénero: las aventuras gráficas.

A partir de la elección del tipo de familia (parejas heterosexuales y homosexuales, elemento que no modifica la forma jugable), el juego nos propone una primera "broma” de marcado orden procedural: es imposible jugar a MonteLab alquilando un piso, solo podemos participar en la experiencia como compradores. Ante la insistencia del jugador por querer alquilar distintos personajes (todos ellos desde la óptica cómico-estereotipada del cuñado, la madre, el amigo listo o la vendedora de la promoción de nuevas viviendas), intentarán convencernos de las virtudes de lo contrario. Si el jugador insiste más, la opción simplemente desaparecerá. En este caso, el modelo procedural se muestra categórico al negar tanto un conjunto de mecánicas (las del alquiler) como sus potenciales dinámicas.

Desde el punto de vista de las mecánicas, MonteLab propone un sistema de elección con afectación en tres variables: la felicidad, el gasto y el valor del inmueble adquirido. Las condiciones de derrota pasan por perder toda la felicidad o arruinarse, mientras que la victoria solo podrá alcanzarse si el jugador vende el piso. Esta primera estructura lúdica ya propone un marco informativo claro: en 2007, comprarse un piso suponía una lucha permanente entre dos factores asimétricos: felicidad y gasto permanente, dadas las circunstancias económicas del momento. Por ello, tras cada elección, el sistema siempre muestra algún tipo de contenido informativo que vincula ficción con eventos reales.

A partir de este marco de reglas, las dinámicas o los comportamientos previstos por el jugador son claros: sobrevivir a la relación causal de nuestras elecciones frente a un conjunto de promesas incumplidas ante la ciudadanía y que el juego insiste en reforzar con distintas fuentes. Así, el juego emerge como una experiencia lúdica de equilibrio y cálculo en el que el ju- 
gador deberá elegir cómo sobrellevar los notables (y crecientes) problemas en materia de sanidad, educación, transporte (especial referencia aquí a las promesas de una estación de AVE), precarización laboral, servicios públicos municipales o la organización de movimientos asociativos como $\mathrm{V}$ de vivienda o la plataforma de afectados por la hipoteca $(\mathrm{PAH})$. En este marco, la relación dinámica con las variables mecánicas del sistema lúdico es claramente alienante: la mayor parte de acciones suelen incrementar el gasto o reducir la felicidad, por lo que las estrategias disponibles para el jugador se tornan cada vez más extremas y, por ende, más desesperadas.

Por ello, no es de extrañar que, desde el punto de vista estético, MonteLab sea un título que establece un pulso permanente con el usuario. Se juega con tensión, impulsando al jugador a un horizonte de irrevocable derrota en el que el sistema económico-social aplasta toda pretensión de supervivencia dentro de su propio marco de concepción. Se trata, por ello, de un juego irresoluble o sin final si se quiere jugar dentro del modelo de la crisis, ya que la concepción dinámica del juego (el juego como sistema que tiende a degradarse tras cada decepción por la decisión inicial de comprar un piso) acaba provocando un colapso mecánico y emocional. Así, la única salida posible y que además está disponible desde su inicio es la venta del hogar.

\section{Guerra a la mentira: la ciudadanía como fuente}

Desde el punto de vista periodístico, Guerra a la mentira constituye un docuweb (una adaptación del reportaje tradicional o documental a las dinámicas de internet) que permite al usuario asumir el rol de investigador a través de fuentes abiertas. Un concepto clave dentro del periodismo digital y en la era del big data. El proyecto parte de un reportaje audiovisual lineal y un documental interactivo. En el reportaje, el usuario descubre a las principales figuras de la verificación de fuentes abiertas como son Bellingcat, con Eliot Higgins al frente, y organizaciones como Amnistía Internacional o Human Rights Watch.

Al igual que ocurría con MonteLab, el primer componente periodístico que se presenta es que Guerra a la mentira está basado en tres casos reales. El primero de ellos es el caso de Omran, el segundo es el del avión que 
en 2014 cayó con 298 civiles en suelo ucraniano y, por último, el ataque que sufrió en octubre de 2016 un convoy humanitario de Naciones Unidas en la localidad siria de Urum al-Kubra.

Una de las principales funciones que tiene la trama es empoderar al ciudadano, para ello el proyecto ofrece recursos para poner en funcionamiento acciones dirigidas al contraste y la verificación de datos que se encuentran en la red. Una finalidad que lo convierte en herramienta clave dentro del fenómeno de las fake news. En la mayor parte de las ocasiones, se trata de informaciones sin fuentes que no pueden contrastarse, pero que muchos ciudadanos asumen como verdaderas por la rapidez con la que se viralizan (Simple Lógica, s. f.). Además, cognitivamente, ante tanta información, el ciudadano no lleva a cabo un procesamiento profundo de toda la amalgama informativa que inunda la red.

Atravesamos un momento en el que la credibilidad de los periodistas y los medios está en juego. Poco a poco, surgen iniciativas como los famosos fact checking para descubrir si una información es verdadera o falsa, pero estas iniciativas no son usadas por la mayoría de los consumidores. Los formatos tradicionales dentro del periodismo tampoco parecen estar a la altura de las circunstancias, porque, en muchas ocasiones, las noticias solo presentan una fuente $y$, a veces, se trata simplemente de fuentes institucionales con intereses muy específicos. Por ello, experimentar con otro tipo de formatos, como hace el equipo del Lab con este proyecto, debería ser la prioridad para los medios de comunicación del presente y futuro.

A través de diversos tutoriales, el usuario aprende a verificar el origen de las imágenes que circulan por la red y los fundamentos de la verificación de vídeo en YouTube, y a buscar noticias en redes sociales, una práctica habitual entre los periodista que tienen que cubrir conflictos en zonas donde no hay medios de comunicación y la única cobertura posible es utilizar a fuentes ciudadanas que se encuentran en el lugar de los hechos.

Según sus autores, el usuario vivirá la experiencia de recrear cómo se utilizan las fuentes abiertas para comprobar la autoría de tres crímenes de guerra. Para ello, formará parte de una conversación en la que, junto con 
otros tres investigadores ficticios, irá recopilando indicios y pruebas para intentar demostrar quiénes son los responsables.

El tratamiento de la información en internet y el acceso a fuentes alternativas a las oficiales o institucionales es un claro ejemplo de las transformaciones que se viven hoy en día en el periodismo. La mediación del periodista entre la fuente primaria y el ciudadano se ha diluido y son los usuarios los que manejan información en bruto a través de fotos, vídeos o textos que se viralizan a velocidad de vértigo y que no han pasado por los filtros correspondientes. El ciudadano asume, a través de este newsgame, el papel de periodista con una vocación de denuncia.

Desde la perspectiva ludológica en Guerra a la mentira, asistimos a un conjunto de mecánicas de juego de claro orden conversacional, cuya forma audiovisual nos remite, inmediatamente, a sistemas de comunicación instantánea como WhatsApp o Telegram. Las reglas predisponen el seguimiento y la resolución de los tres casos descritos mediante el aprendizaje de distintas técnicas y métodos de obtención de fuentes y verificación de la información oficial. Al final de cada caso, pequeños minijuegos (contrastar fotografías sobre el terreno con fotografías satélite, relacionar tuits con la ubicación de misiles BUK y detectar el impacto de determinadas armas en camiones e infraestructuras) refuerzan la experiencia lúdico-espectatorial con una aplicación práctica para cada uno de los casos de estudio. Por ello, junto con el modelo conversacional lineal, estos pequeños minijuegos de puzles nos remiten claramente al género de aventuras y, específicamente, al subgénero de los títulos hidden objects.

Las mecánicas específicas del juego como sistema se limitan, excepción hecha al minijuego final, a elecciones arbóreas o ramificadas que dotan a la experiencia de mayor profundidad. Cada nodo o alternancia de elección suele acompañarse con un conjunto de tuits o diversos vídeos de fuentes reales que colaboran activamente en el devenir narrativo del juego. Por ello, esta forma propia de la ficción interactiva debe entenderse, además, como un modelo reglado que explota con interés y eficacia las distintas ventanas que proporciona el relato transmedia de cada caso. 
De este modo, las dinámicas se enmarcan en la actualidad social de la mensajería instantánea y se centran, esencialmente, en desarrollar estrategias de comprensión de las distintas preguntas que proponen cada uno de los casos de estudio. En este sentido, las elecciones no son tan significativas en su manifestación performativa (es decir, por su impacto en el sistema de juego como una toma de decisión activa del jugador) como por su contribución a expandir y profundizar el diálogo que establece en la resolución y en el contraste de fuentes. Por ello, Guerra a la mentira es un título estrictamente story driven, es decir, es un juego más relevante por la historia que despliega y guía las acciones del jugador que por un potencial entramado de complejas estrategias y reglas subyacentes.

En cuanto al nivel estético, la propuesta lúdica fomenta la experiencia del "juego de detectives" y, más concretamente, la tensión humanitaria que existe entre los extremos "verdad" y "mentira”. Es en este eje en el que el juego desarrolla su expresión emocional, sin caer en banalizaciones ni discursos dogmáticos, y despierta el interés en el jugador por el rol que tiene la búsqueda y el contraste de la información en situaciones de urgencia humanitaria. De este modo, Guerra a la mentira defiende la búsqueda de la verdad como valor supremo y convierte su búsqueda en su mayor expresión lúdica y estética.

\section{Conclusiones}

En relación con MonteLab, se parten de unos hechos reales para mostrar un problema social con alto interés público. Cumple todos los criterios de noticiabilidad y, además, utiliza un conjunto de fuentes numerosas y plurales que supera a las que habitualmente se utilizan para, por ejemplo, un reportaje periodístico tradicional. En este enmarcado informativo, la dimensión lúdica de MonteLab propone un juego de imposible resolución en su base experiencial al oponer dos variables: felicidad y gasto, que son, desde la perspectiva de las mecánicas, irresolubles. De este modo, la relación entre información y juego en MonteLab resuelve el doble objetivo del diseño de un newsgame: contextualiza, documenta e informa sobre una realidad $y$, a su vez, hace partícipe al usuario de los problemas sociales subyacentes al sistema lúdico y periodístico. 
En cuanto a Guerra a la mentira, se pone de relieve, a través de casos reales, el proceso de verificación y contraste de las fuentes abiertas en internet en zonas de conflicto. El usuario asume, de alguna forma, el rol de periodista durante el transcurso de una investigación de la que se sirve de diversas herramientas accesibles y abiertas que empoderan al ciudadano. Se trata de un proyecto que puede servir como base para que el usuario aprenda a detectar en la red falsas noticias y sea capaz de chequear los datos aportados en ellas para comprobar su veracidad.

Los elementos y recursos que se facilitan al usuario en este newsgame responden a la función didáctica de este género emergente y, desde la óptica ludológica, la experiencia de juego se inscribe en el marco transmedia posible gracias a los modelos actuales de mensajería instantánea. Junto con el rastreo story driven de las fuentes, el juego ancla su sentido retórico-procedural al establecer un enlace directo entre lo descubierto (información) y lo maleable (interacción) de los minipuzles que muestran cómo las mentiras pueden desacreditarse cuando se cuenta con las fuentes apropiadas.

El análisis de ambos casos conlleva, a su vez, un conjunto de conclusiones más generales que son de gran interés. Por un lado, el newsgame contemporáneo no se limita a participar de manera pasiva en un ecosistema interactivo informativo (algo que pudiera interpretarse ante la homogeneidad transmediática y la convergencia estrictamente tecnológica de Jenkins), sino que, tal y como ya apuntó desde el plano teórico Sicart (2016), su principal aportación y novedad radica, precisamente, en empoderar al jugador como ciudadano, y hacerlo partícipe de las soluciones a los problemas que la información pone encima de la mesa. De este modo, el newsgame actual amplía el discurso periodístico y complementa su función informativa con un espacio de soluciones sociales y cambios posibles en la esfera pública de acción. Así, el newsgame empodera tanto al jugador como al periodista digital y convierte a ambos en actores clave del debate presente en la opinión pública.

Por otro lado, el docuweb ha conseguido superar un cierto estaticismo hipertextual para adentrarse en funciones mucho más proactivas en relación con el usuario y con la construcción de la experiencia interactiva. 
La eclosión del big data, la relevancia de las fuentes en un contexto digital y el marcado carácter social del género documental lo convierten en un formato muy a considerar cuando la principal perspectiva periodística sobre el objeto radica en la configuración del relato en sí misma y el impacto que determinada toma de decisiones suele tener en la realidad fáctica plasmada en el trabajo periodístico antes de la creación del docuweb. De este modo, y en paralelo a lo expresado desde la generalidad del newsgame, el docuweb emerge como una poderosa síntesis entre la información social propia del género en el medio pasivo y su vínculo con una interacción proactiva y empoderadora propia del contexto digital.

\section{Referencias}

Aston, J. \& Gaudenzi, S. (2012). Interactive documentary: Setting the field. Studies in Documentary Film, 6(2), 125-139. https://www.tandfonline.com/doi/abs/10.1386/sdf.6.2.125_1

Bardin, L. (1996). Análisis de contenido. Madrid, España: Akal.

Bogost, I. (2007). Persuasive games: The expressive power of videogames. Massachusetts, EE. UU.: MIT Press.

Bogost, I., Ferrari S. \& Schweizer, B. (2012). Newsgames: Journalism at play. Massachusetts, Estados Unidos: MIT Press.

Brathwaite, B. \& Sharp, J. (2010). The mechanic is the message: A post mortem in progress. En K. Schrier \& D. Gibson (Eds.), Ethics and game design: Teaching values through play (pp. 311-329). Pennsylvania, EE. UU.: IGI Global.

Carrera Álvarez, P., Limón Serrano, N., Herrero Curiel, E. y Sainz de Baranda Andújar, C. (2013). Transmedialidad y ecosistema digital. Historia y Comunicación Social, 18, 535-545. https://doi.org/10.5209/ rev_HICS.2013.v18.44257 
Celeste Marrocco, M. (2013). De espectador cinematográfico a interactor: Art games y newsgames, expresiones artísticas en el siglo XXI. Toma Uno, 2, 153-165. http://publicaciones.ffyh.unc.edu.ar/index.php/toma1/index

Domínguez-Martín, E. (2015). Periodismo inmersivo o cómo la realidad virtual y el videojuego influyen en la interfaz e interactividad del relato de actualidad. El Profesional de la Información, 24(4), 413423. https://doi.org/10.3145/epi.2015.jul.08

Ferrari, P. (2010). A força da mídia social interface e linguagem jornalística no ambiente digital. São Paulo, Brasil: Factash.

Flanagan, M. (2009). Critical play: Radical game design. Massachussets, EE. UU.: The MIT Press.

Gifreu Castells, A. (2013). El documental interactivo: evolución, caracterización y perspectivas de desarrollo. Barcelona, España: UOC.

Gómez García, S. y Cabeza San Deogracias, J. (2016). El discurso informativo de los newsgames: el caso Bárcenas en los juegos para dispositivos móviles. Cuadernos.info, 38, 137-148. https://doi. org/10.7764/cdi.38.593

Gómez García, S. y Navarro Sierra, N. (2013). Videojuegos e información: una aproximación a los newsgames españoles como nueva óptica informativa. Icono 14, Revista de comunicación y tecnologías emergentes, 11(2), 31-51. https://doi.org/10.7195/ri14.v11i2.604

Herrero Curiel, E. (2014). Del 11M al 15M: periodistas y redes sociales en España. Barcelona, España: UOC.

Hunicke, R., Leblanc, M. \& Zubek, R. (2004). MDA: A formal approach to game design and game research. En Proceedings of the AAAI Workshop on Challenges in Game AI. Nineteenth National Conference of Artificial Intelligence. San José, EE. UU.: AAAI Press. 
Jenkins, H. (2008). Convergence culture: la cultura de la convergencia de los medios de comunicación. Barcelona, España: Paidós.

Jones, S. (2017). Disrupting the narrative: Immersive journalism in virtual reality. Journal of Media Practice, 18(2-3), 171-185. https:// doi.or $\mathrm{g} / 10.1080 / 14682753.2017 .1374677$

Kazez, R. (2009). Los estudios de casos y el problema de la selección de la muestra: aportes del sistema de matrices de datos. Subjetividad y Procesos Cognitivos, 13(1), 71-89.http://dspace.uces.edu.ar:8180/ xmlui/handle/123456789/727

Lippman, W. (2003). La opinión pública. Madrid, España: Langre.

López Hidalgo, A. y Ufarte Ruiz, M. J. (2016). Laboratorios de periodismo en España: nuevas narrativas y retos de futuro. Ámbitos: revista internacional de comunicación, 34. https://idus.us.es/xmlui/handle/11441/60784

Martínez Arias, S. y Díaz Arias, R. (2016). Pautas y metodología para la creación de un nuevo género periodístico. ZER: Revista de Estudios de Comunicación, 21(41), 71-91.https://doi.org/10.1387/zer.17263

Martínez Cano, F. J. (2016). Newsgames: nuevas tendencias en el periodismo ibérico. CoSECivi, 1, 159-169. https://pdfs.semanticscholar. org/7358/1bc47132dfebf39b678620df6b747d7864b7.pdf

Moloney, K. T. (2011). Porting transmedia storytelling to journalism (Tesis de maestría, University of Denver, Denver, EE. UU.).

Murray, J. H. (1998). Hamlet on the holodeck: The future of narrative in cyberspace. Nueva York, EE. UU.: The Free Press.

Ryan, M.-L. (Ed.) (2004). Narrative across media: The languages of storytelling. Lincoln, EE. UU.: University of Nebraska Press. 
Salen, K. \& Zimmerman, E. (2004). Rules of play: Game design fundamentals. Massachusetts, EE. UU.: MIT Press.

Samson, E. (2015). Informar con juegos serios: sus potencialidades y limitaciones para el periodismo actual. Post (S), 1(1), 121-149. https:// doi.org/10.18272/posts.v1i1.239

Sicart, M. (2008). Newsgames: Theory and design. En S. M. Stevens \& S. J. (Eds.), Entertainment Computing-ICEC 2008: 7th International Conference, Pittsburgh, PA, USA, September 25-27, 2008, Proceedings (pp. 27-33). Berlín, Alemania: Springer.

Sicart, M. (2011). Against procedurality. Game Studies, 11(3). http://gamestudies.org/1103/articles/sicart_ap?utm_source=tech.mazavr.tk\&utm_medium=link\&utm_compaign=article

Simple Lógica. (s. f.). I Estudio sobre el Impacto de las Fake News en Espana. https://d3vjcwm65af87t.cloudfront.net/novacdn/EstudioPescanova.pdf 


\section{Anexo 1. Tabla de análisis}

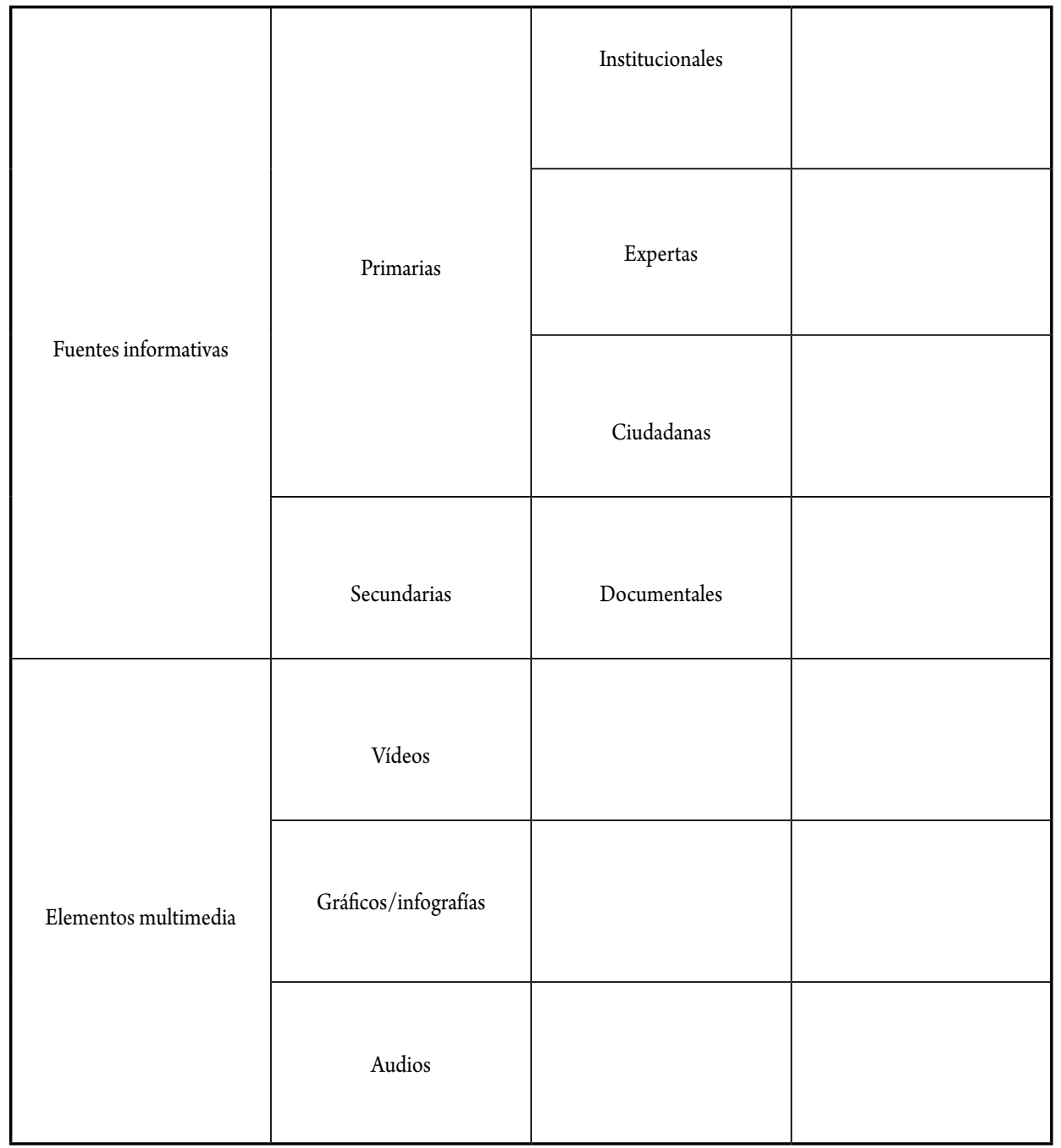

